\title{
Java PathFinder \\ A Translator from Java to Promela
}

\author{
Klaus Havelund \\ NASA Ames Research Center \\ Recom Technologies \\ Moffett Field, CA, USA \\ havelundoptolemy .arc.nasa.gov \\ http://ase.arc.nasa.gov/havelund
}

JAVA PATHFinder [2], JPF, is a prototype translator from JaVA to Promela, the modeling language of the SPIN model checker [4]. JPF is a product of a major effort by the Automated Software Engineering group at NASA Ames to make model checking technology part of the software process. Experience has shown that severe bugs can be found in final code using this technique [1], and that automated translation from a programming language to a modeling language like Promela can help reducing the effort required.

JPF allows a programmer to annotate his JAVA program with assertions and verify them using the SPIN model checker. In addition, deadlocks can be identified. An assertion is written as a call to an assert method defined in a predefined JAVA class, the Verify class. The argument to the method is a boolean JAVA expression over the state variables. The Verify class contains additional temporal logic methods which allow to state temporal logic properties about static variables. Hence JAVA itself is used as the specification language. An application of JPF is described elsewhere in the proceedings [3].

A respectable subset of $\mathrm{J}_{\mathrm{AVA}}$ is covered by JPF, including dynamic object creation, object references as first class citizens, inheritance, exceptions, interrupts, and perhaps most importantly: thread operations. Among major concepts not translated are: packages, method overloading and overriding, method recursion, strings, and floating point numbers. Finally, the class library is not translated.

\section{References}

1. K. Havelund, M. Lowry, and J. Penix. Formal Analysis of a Space Craft Controller using SPIN. In G. Holzmann, E. Najm, and A. Serhrouchni, editors, Proceedings of the th SPIN workshop, Paris, France, November 1998. To appear in IEEE Transactions of Software Engineering.

2. K. Havelund and T. Pressburger. Model Checking Java Programs using Java PatbFinder. Appearing in International Joumal on Software Tools for Technology Transfer (STTT), 1999.

3. K. Havelund and J. Skakkebak. Applying Model Checking in Java Verification. In R. Gerth, G. Holzmann, and S. Leue, editors, Proceedings of the 6th SPIN workshop (these proceedings), Toulouse, France, September 1999.

4. G. Holzmann. The Design and Validation of Computer Protocols. Prentice Hall, 1991. 




\section{Retroreflective Focusing Schlieren (RFS) Image of Jet in Wind Tunnel}

April 6, 1992,

J.T. Heineck NASA Ames Research Center

Steven Jaeger, Sterling Software

Situation: A high pressure air jet from a table top is blown upwards into a cross flow. The retroreflective material and source grid were placed outside the test section behind a window. The light source, camera, beam splitter and cutoff grid are mounted on the other side of the wind tunnel. Light source was a xenon flash with a duration of I msec Conditions: Jet Mach number was 1.(1)7, the wind tunnel velocity was 170 miles per hour, wind tunnel temperature: $78.9^{\circ} \mathrm{F}$.

Image by J.T. Heineck, NASA Ames Research Center. 
For publication in an upcoming book titled "Flow Visualization: Techniques and Examples" published by the Imperial College Press.

Reference: Photograph: "Retroreflective Focussing Schlieren (RFS) Image of a Jet in Wind Tunnel" 